\title{
The in vitro effect of xylitol on chronic rhinosinusitis biofilms*
}

\author{
Ravi Jain', Tony Lee', Timothy Hardcastle', Kristi Biswas', Fiona Radcliff², \\ Richard Douglas
}

Department of Surgery, The University of Auckland, Grafton, Auckland, New Zealand

2 Department of Molecular Medicine and Pathology, The University of Auckland, Grafton, Auckland, New Zealand
Rhinology 54: 323-328, 2016

DOI:10.4193/Rhino15.380

${ }^{*}$ Received for publication:

December 21, 2015

Accepted: April 27, 2016

\begin{abstract}
Introduction: Biofilms have been implicated in chronic rhinosinusitis (CRS) and may explain the limited efficacy of antibiotics. There is a need to find more effective, non-antibiotic based therapies for CRS. This study examines the effects of xylitol on CRS biofilms and planktonic bacteria.
\end{abstract}

Methods: Crystal violet assay and spectrophotometry were used to quantify the effects of xylitol (5\% and $10 \%$ solutions) against Staphylococcus epidermidis, Pseudomonas aeruginosa, and Staphylococcus aureus. The disruption of established biofilms, inhibition of biofilm formation and effects on planktonic bacteria growth were investigated and compared to saline and no treatment.

Results: Xylitol $5 \%$ and $10 \%$ significantly reduced biofilm biomass (S. epidermidis), inhibited biofilm formation (S. aureus and $P$. aeruginosa) and reduced growth of planktonic bacteria (S. epidermidis, S. aureus, and P. aeruginosa). Xylitol 5\% inhibited formation of S. epidermidis biofilms more effectively than xylitol $10 \%$. Xylitol $10 \%$ reduced S. epidermidis planktonic bacteria more effectively than xylitol 5\%. Saline, xylitol 5\% and 10\% disrupted established biofilms of S. aureus when compared with no treatment. No solution was effective against established $P$. aeruginosa biofilm.

Conclusions: Xylitol has variable activity against biofilms and planktonic bacteria in vitro and may have therapeutic efficacy in the management of CRS.

Key words: chronic rhinosinusitis, xylitol, biofilms, bacteria, therapeutic irrigation

\section{Introduction}

Bacterial biofilms have been implicated in chronic rhinosinusitis (CRS) pathogenesis and disease recalcitrance following endoscopic sinus surgery (ESS) ${ }^{(1-3)}$. Biofilm positive patients have been shown to be more likely to need surgical intervention, worse postoperative symptoms, persistent inflammation and recurrent infections ${ }^{(4-9)}$.

Targeted treatment of sinonasal biofilms with long-term efficacy has not yet been described. Systemic antibiotics such as vancomycin, ciprofloxacin, piperacillin and ceftazidime have been shown to be ineffective at standard concentrations ${ }^{(10,11)}$. Topical use of mupirocin has been shown to reduce biofilm mass but is associated with high microbiological failure rate in patients with surgically recalcitrant CRS ${ }^{(12)}$. As rhinosinusitis now accounts for more than $11 \%$ of all antibiotic prescriptions in the United States, a non-antibiotic anti-biofilm agent that is inexpensive, effective and safe is required ${ }^{(1,13)}$.

Xylitol is a five-carbon sugar alcohol, which occurs naturally in many fruits and vegetables, and is used widely in the food industry as a sweetener. Recently, it has gained more popularity as an anti-biofilm agent, particularly in the field of periodontics ${ }^{(14)}$. It has been shown to improve CRS symptoms when administered as a sinus lavage solution ${ }^{(15)}$.

The aim of this study was to assess the effect of xylitol in vitro on established biofilms, biofilm formation and planktonic bacteria 
that were either derived from CRS patients or commercial strains of known sinonasal pathogens. Furthermore, this study aimed to determine xylitol's potential as an adjunct to topical therapy.

\section{Materials and methods}

All procedures were undertaken at the School of Biological Sciences and Faculty of Medicine and Health Sciences of The University of Auckland, following approval with national and institutional human research ethics committees.

\section{Bacterial isolates}

Two sterile rayon-tipped swabs (Copan, Murrieta, CA, USA. \#170KS01) were taken from the right and left middle meatuses of two patients with CRS undergoing endoscopic sinus surgery. Swabs were taken following general anesthesia and prior to administration of intravenous antibiotics. These were placed in sterile Eppendorf tubes and transported on ice for immediate culturing on nutrient and Columbia horse blood agar (Fort Richard Laboratories Ltd, New Zealand) for 48 hours at $37^{\circ} \mathrm{C}$.

Fourteen bacterial strains were isolated and found amenable to culture in tryptic soy broth (TSB) (Difco Laboratories, Sparks, MD, USA). These strains were investigated further for their biofilm forming capacity. Broth from each isolate $(200 \mu \mathrm{L})$ was pipetted in triplicate into wells of a flat-bottomed 96-well microtiter plate (Thermo Fisher Scientific Inc, New Zealand), which had been treated for tissue culture to optimise bacterial attachment to well surfaces ${ }^{(16)}$. Negative control wells were instilled with broth media only. Plates were incubated in static conditions for $24 \mathrm{~h}$ at $37^{\circ} \mathrm{C}$ to allow biofilm formation and attachment to well surfaces. After incubation the wells were aspirated and washed with phosphate buffered saline to remove planktonic and nonadherent bacteria ${ }^{(16)}$.

A crystal violet assay with optical density measured at 570 nm (Perkin Elmer Enspire Multimode Plate Reader, Waltham, MA, USA) was used to quantify biofilm biomass as previously described ${ }^{(16-18)}$. Biofilm formation from broth and subsequent optical density measurement was repeated four times. The most reliable and greatest biomass forming isolates were identified by matrix-assisted laser desorption/ionisation time of flight (MALDITOF) mass spectrometry (Bruker UltrafleXtreme, Billerica, MA, USA) and were all found to be S. epidermidis, of which the best biofilm-forming isolate was chosen for use in this study.

In addition, commercial biofilm-forming isolates of $S$. aureus New-man and P. aeruginosa PAO1 (Institute of Infection, Immunity and Inflammation, University of Nottingham, UK) were also obtained for this study. Biofilm growth was optimised for S. aureus and $P$. aeruginosa by addition of $1 \%(\mathrm{w} / \mathrm{v})$ glucose to overnight broth cultures diluted 1:100 in fresh TSB $(15 \mathrm{~mL})$.

\section{Treatment groups}

Xylitol (Langdon ingredients, Melbourne, Australia) was obtained in powdered form and diluted with TSB to obtain $5 \%$ $(\mathrm{w} / \mathrm{v})$ and $10 \%(\mathrm{w} / \mathrm{v})$ solutions. These were compared with a $0.9 \%$ $(\mathrm{w} / \mathrm{v})$ solution of $\mathrm{NaCl}$ and a TSB only group, which served as a negative control.

\section{Disruption of established biofilms}

Biofilms of S. epidermidis, S. aureus, and P. aeruginosa were grown on 96-well plates as described. Each bacterial strain was grown in replicates of six for each treatment group. Following incubation and washing, $200 \mu \mathrm{L}$ of each treatment solution was added to the six wells corresponding to each group. Plates were covered with a lid and treated for one hour at room temperature in static conditions. After the treatment period, each well was washed three times with $200 \mu \mathrm{L}$ of phosphate buffered saline (PBS) to remove planktonic and non-adhering bacteria. Biofilms were stained and measured using crystal violet assay. This experiment was repeated four times for each bacterial strain.

Inhibition of biofilm formation

In this study, biofilms were grown on plates in the presence of each of the treatment solutions. In each well, high concentration treatment solutions $(50 \mu \mathrm{L})$ were added to overnight broth culture for each isolate $(200 \mu \mathrm{L})$ to makeup the working treatment solutions. Each treatment consisted of six replicates. Plates were incubated for $24 \mathrm{~h}$ at $37^{\circ} \mathrm{C}$ to allow biofilms to form. Wells were washed with PBS and underwent crystal violet assay. This experiment was repeated four times for each bacterial strain.

\section{Effects on planktonic bacteria}

To assess the effects of treatment on planktonic bacteria, TSB with treatment solutions $(100 \mu \mathrm{L})$ were added to bacteria grown to mid-exponential phase in TSB $(100 \mu \mathrm{L})$. Each treatment consisted of six replicates. Plates were incubated at $37^{\circ} \mathrm{C}$ for four hours. Spectrophotometry was used to measure absorbance at 600 $\mathrm{nm}$ and compared to similar treatment TSB solutions without bacterial culture. This experiment was repeated three times for each bacterial strain.

\section{Statistical analysis}

Statistical analyses were performed using SPSS (Version 22; IBM, New York, nY, USA). All results were described using mean difference $(\Delta) \pm$ standard error. Groups were compared using a one-way analysis of variance (ANOVA) with post-hoc Tukey's HSD to adjust for multiple comparisons. Significance was accepted when $P$ was less than 0.05 .

\section{Results}

Disruption of established biofilms (Figure 1). Staphylococcus epidermidis 

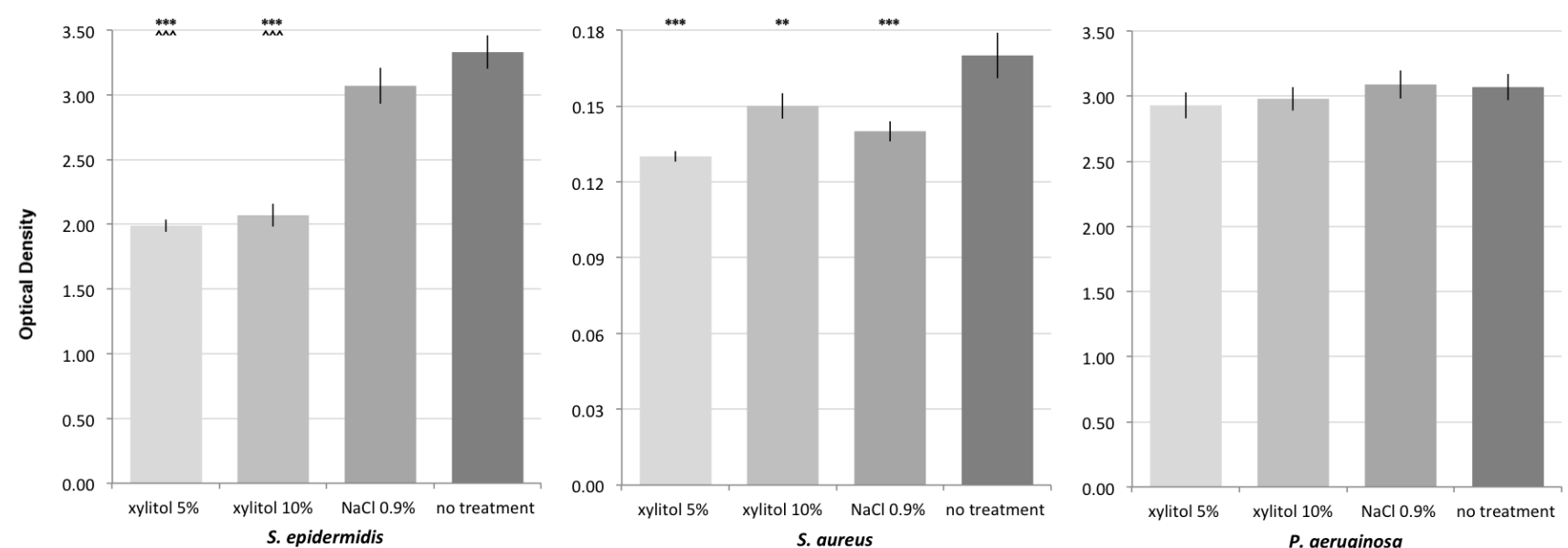

Figure 1. Disruption study: effects of solutions on the optical density of established biofilms after 1 hour of treatment.

$\mathrm{n}=24$ for each bacteria and treatment combination. Error bars represent standard error of the mean. Significance: ${ }^{*} P<0.05$, ${ }^{* * P}<0.01$ and ${ }^{* * *} P<0.001$ compared to no treatment, $\wedge P<0.05, \wedge \wedge P<0.01$ and $\wedge \wedge \wedge P<0.001$ compared to saline.
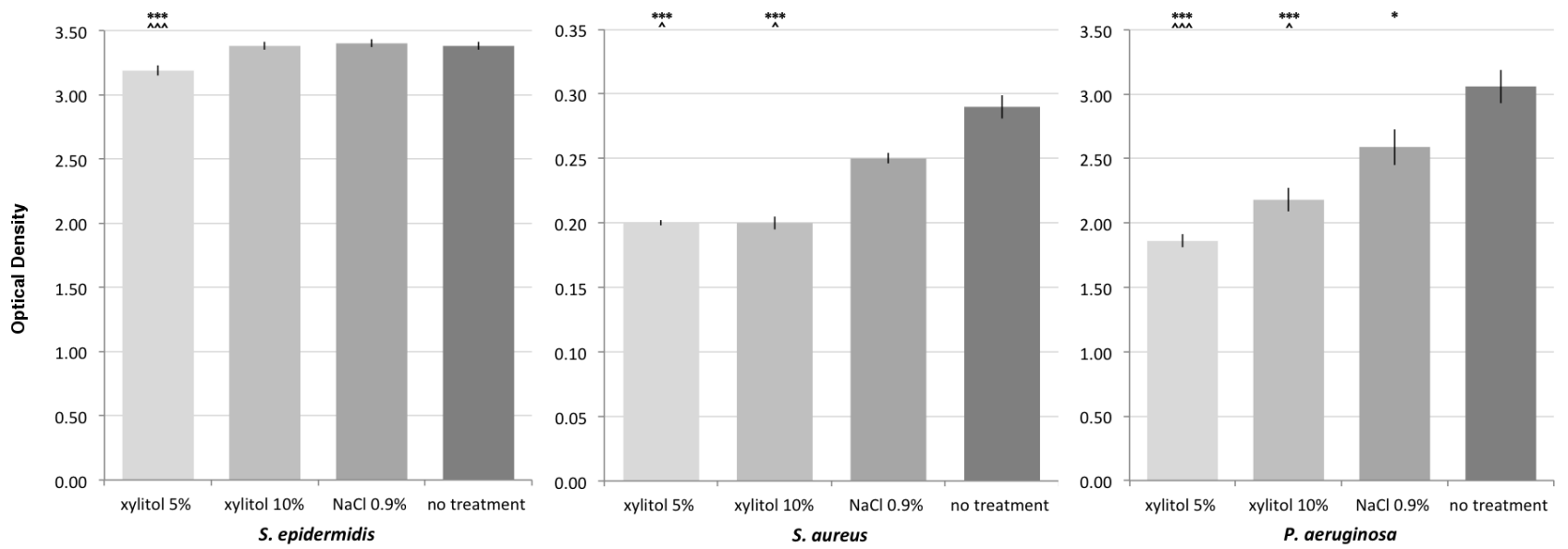

Figure 2. Inhibition study: optical density of bacterial biofilms formed in the presence of solutions after 24 hours.

$\mathrm{n}=24$ for each bacteria and treatment combination. Error bars represent standard error of the mean. Significance: ${ }^{*} \mathrm{P}<0.05,{ }^{* *} \mathrm{P}<0.01$ and ${ }^{* * *} \mathrm{P}<0.001$ compared to no treatment, $\wedge P<0.05, \wedge \wedge P<0.01$ and $\wedge \wedge \wedge P<0.001$ compared to saline.

Significant reductions in biofilm biomass were observed with xylitol $5 \%(\Delta 1.3 \pm 0.1, \mathrm{P}<0.001)$ and xylitol $10 \%(\Delta 1.3 \pm 0.1$, $\mathrm{P}<0.001)$ when compared with no treatment. Both the $5 \%$ and $10 \%$ solutions were also significantly more effective than saline $(\Delta 1.1 \pm 0.1, \mathrm{P}<0.001$ and $\Delta 1.0 \pm 0.1, \mathrm{P}<0.001)$. There was no difference between xylitol $5 \%$ and $10 \%$.

\section{Staphylococcus aureus}

Reductions of biomass were seen with xylitol $5 \%(\Delta 0.44 \pm 0.01$, $\mathrm{P}<0.001)$, xylitol $10 \%(\Delta 0.29 \pm 0.01, \mathrm{P}<0.004)$ and saline $(\Delta 0.31$ $\pm 0.01, P<0.001)$ when compared with no treatment. No differences were seen between the xylitol solutions and saline.

\section{Pseudomonas aeruginosa}

No significant differences were seen between the experimental groups.
Inhibition of biofilm formation (Figure 2).

\section{Staphylococcus epidermidis}

Only xylitol $5 \%$ was shown to significantly inhibit S. epidermidis biofilm formation when compared to no treatment $(\Delta 0.19 \pm$ $0.05, \mathrm{P}<0.001)$, saline $(\Delta 0.21 \pm 0.05, \mathrm{P}<0.001)$ and $x y$ litol $10 \%(\Delta$ $0.18 \pm 0.05, P<0.001)$. There was no difference between saline and no treatment groups.

\section{Staphylococcus aureus}

Inhibition of biofilm formation was seen with both xylitol 5\% and xylitol $10 \%$ when compared with no treatment $(\Delta 0.09 \pm$ $0.02, \mathrm{P}<0.001$ and $\Delta 0.09 \pm 0.02, \mathrm{P}<0.001)$ and saline $(\Delta 0.05 \pm$ $0.02, \mathrm{P}=0.03$ and $\Delta 0.06 \pm 0.02, \mathrm{P}<0.03)$. There was no difference between preparations of xylitol or between saline and no treatment groups. 

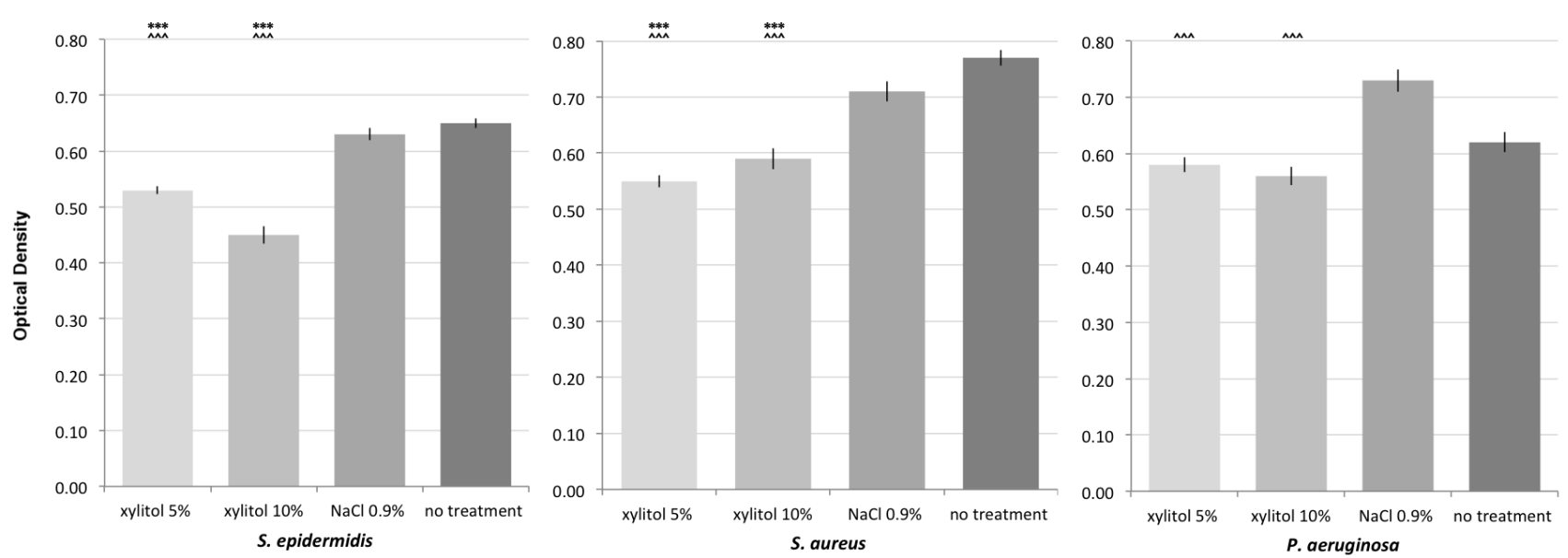

Figure 3. Planktonic study: optical density of planktonic bacteria in the presence of solutions after 4 hours.

$\mathrm{n}=18$ for each bacteria and treatment combination. Error bars represent standard error of the mean. Significance: ${ }^{*} \mathrm{P}<0.05,{ }^{* *} \mathrm{P}<0.01$ and ${ }^{* * *} \mathrm{P}<0.001$ compared to no treatment, $\wedge \mathrm{P}<0.05, \wedge \wedge \mathrm{P}<0.01$ and $\wedge \wedge \wedge \mathrm{P}<0.001$ compared to saline.

\section{Pseudomonas aeruginosa}

Xylitol 5\%, xylitol 10\% and saline were able to inhibit P. aeruginosa biofilm formation when compared to no treatment $(\triangle 1.2 \pm$ $0.15, \mathrm{P}<0.001, \Delta 0.88 \pm 0.15, \mathrm{P}<0.001$ and $\Delta 0.47 \pm 0.15, \mathrm{P}<0.02)$. Xylitol $5 \%$ and $10 \%$ were also both more efficacious than saline $(\Delta 0.73 \pm 0.15, \mathrm{P}<0.001$ and $\Delta 0.41 \pm 0.15, \mathrm{P}=0.04)$, however there was no significant difference between the two preparations.

\section{Effects on planktonic bacteria (Figure 3).}

\section{Staphylococcus epidermidis}

Solutions of both xylitol 5\% and 10\% significantly inhibited planktonic $S$. epidermidis when compared with no treatment $(\Delta 0.12 \pm 0.02, \mathrm{P}<0.001$ and $\Delta 0.20 \pm 0.02, \mathrm{P}<0.001)$ and saline $0.9 \%(\Delta 0.1 \pm 0.02, \mathrm{P}<0.001$ and $\Delta 0.18 \pm 0.02, \mathrm{P}<0.001)$. When compared, xylitol $10 \%$ was more effective than xylitol $5 \%(\Delta 0.08$ $\pm 0.02, \mathrm{P}<0.001)$.

\section{Staphylococcus aureus}

Solutions of both xylitol $5 \%$ and $10 \%$ again significantly inhibited planktonic S. aureus when compared with no treatment $(\Delta$ $0.22 \pm 0.02, \mathrm{P}<0.001$ and $\Delta 0.18 \pm 0.02, \mathrm{P}<0.001)$ and saline $0.9 \%$ $(\Delta 0.16 \pm 0.02, \mathrm{P}<0.001$ and $\Delta 0.12 \pm 0.02, \mathrm{P}<0.001)$. When compared, there was no difference between xylitol $5 \%$ and $10 \%$.

\section{Pseudomonas aeruginosa}

Solutions of both xylitol $5 \%$ and $10 \%$ significantly inhibited planktonic $P$. aeruginosa when compared with saline $0.9 \%(\Delta$ $0.15 \pm 0.02, \mathrm{P}<0.001$ and $\Delta 0.16 \pm 0.02, \mathrm{P}<0.001)$. There was no difference between xylitol $5 \%$ and $10 \%$.

\section{Discussion}

This study has examined the effects of xylitol on three sinonasal bacterial species, S. epidermidis, S. aureus, and P. aeruginosa, that are capable of forming biofilms.

Xylitol is an inexpensive and sweet tasting sugar alcohol (or polyol), which has been widely used in the food industry to replace sucrose. More recently xylitol has gained popularity for its antimicrobial effects ${ }^{(14,19,20)}$. Xylitol is thought to exert its antibacterial activity by a number of mechanisms including reduction of the salt concentration of airway surface liquid, repressing bacterial metabolism of glucose to lactate, and disruption of the biofilm extracellular matrix ${ }^{(21-25)}$.

In this study, xylitol was variously active against the three examined microorganisms. When compared with saline, both xylitol $5 \%$ and $10 \%$ were able to significantly reduce biofilm biomass (S. epidermidis), inhibit biofilm formation (S. aureus and $P$. aeruginosa) and reduce planktonic bacteria (S. epidermidis, $S$. aureus and $P$. aeruginosa).

Generally, a higher concentration of xylitol was not more effective than the isosmotic $5 \%$ solution. Differences in activity between the concentrations of xylitol were only seen for inhibition of S. epidermidis biofilms (in which $5 \%$ was significantly more effective) and against S. epidermidis planktonic bacteria (in which $10 \%$ was significantly more effective).

By its in vitro design, the results of this study are limited and may not directly translate to the in vivo effects of xylitol. For example, no solution in this study was effective against established $P$. aeruginosa. Established biofilms in this study were exposed to treatments for one hour. This may not be enough time for xylitol to completely penetrate bacterial membranes. Previous studies assessing the effect of xylitol on $P$. aeruginosa over a $24 \mathrm{~h}$ period have suggested that the addition of a syner- 
gist such as lactoferrin increases permeability of bacterial membranes and structural disruption of biofilms ${ }^{(26,27)}$. In vivo, the ability of xylitol to remain on the apical surfaces of respiratory epithelia and lower the airway surface liquid salt concentration without being metabolised by $P$. aeruginosa might increase the antimicrobial effect ${ }^{(22,28)}$.

The length of exposure time of biofilms to xylitol in in vitro studies may be longer than exposure in the paranasal sinuses following nasal lavage, where it is not known how much xylitol would remain in the cavities, or for what duration. The hydrodynamic nature of nasal lavage may also have an effect on biofilm disruption and was not evaluated in this study. Despite this, a pilot randomised control study using a once daily xylitol $5 \%$ rinse observed a significant reduction in SNOT-20 scores when compared to saline ${ }^{(15)}$.

Overall, this study has demonstrated the activity of xylitol against three commonly found sinonasal bacteria in both biofilm and planktonic state, which reaffirms its previously described antimicrobial effects. The therapeutic potential of xylitol lies in its utility as a safe, inexpensive, well tolerated, non-antibiotic topical treatment for CRS ${ }^{(15)}$. As a nasal irrigation it may have a stronger regulatory effect on the sinonasal microbiota than conventionally used saline and have a particular role in the post surgical sinus cavities where control of biofilms may influence clinical outcomes. However, the effects of xylitol on the microbiota in vivo has not yet been established and the effects could be different in the context of a more complex interaction between multiple bacterial populations and innate immune factors, including the airway surface liquid.

\section{Conclusion}

Xylitol has reliable antimicrobial activity against established biofilms, inhibiting biofilm formation and reducing planktonic bacteria in vitro. In this study, xylitol was almost universally more effective than saline. Xylitol is a safe and non-antibiotic potential for topical therapy in CRS and deserves strong consideration for further patient based studies.

\section{Authorship contribution}

RD: Principal investigator, study design and manuscript review. RJ: Study design, data collection, analysis and manuscript writing. TL, TH: Data collection, analysis and manuscript review. KB, FR: Collaborating investigators, manuscript review.

\section{Conflict of interest}

None.

\section{References}

1. Jain R, Douglas R. When and how should we treat biofilms in chronic sinusitis? Curr Opin Otolaryngol Head Neck Surg. 2014; 22:16-21.

2. Danielsen KA, Eskeland O, Fridrich-Aas K, Orszagh VC, Bachmann-Harildstad G, Burum-Auensen E. Bacterial biofilms in patients with chronic rhinosinusitis: a confocal scanning laser microscopy study. Rhinology. 2014; 52:150-5.

3. Psaltis AJ, Ha KR, Beule AG, Tan LW, Wormald P-J. Confocal scanning laser microscopy evidence of biofilms in patients with chronic rhinosinusitis. Laryngoscope. John Wiley \& Sons, Inc; 2007; 117:1302-6.

4. Tan NC-W, Foreman A, Jardeleza C, Douglas $\mathrm{R}$, Tran H, Wormald P-J. The multiplicity of Staphylococcus aureus in chronic rhinosinusitis: correlating surface biofilm and intracellular residence. Laryngoscope. 2012; 122:1655-60.

5. Zhang Z, Han D, Zhang S, Han Y, Dai W Fan $E$, et al. Biofilms and mucosal healing in postsurgical patients with chronic rhinosinusitis. Am J Rhinol Allergy. 2009; 23:506-11.

6. Psaltis AJ, Weitzel EK, Ha KR, Wormald P-J. The effect of bacterial biofilms on postsinus surgical outcomes. Am J Rhinol. 2008; 22:1-6.

7. Singhal D, Foreman A, Jervis-Bardy J, Bardy J-J, Wormald P-J. Staphylococcus aureus biofilms: Nemesis of endoscopic sinus sur- gery. Laryngoscope. 2011; 121:1578-83.

8. Singhal D, Psaltis AJ, Foreman A, Wormald P-J. The impact of biofilms on outcomes after endoscopic sinus surgery. Am J Rhinol Allergy. OceanSide Publications, Inc; 2010; 24:169-74.

9. Bendouah Z, Barbeau J, Hamad WA, Desrosiers M. Biofilm formation by Staphylococcus aureus and Pseudomonas aeruginosa is associated with an unfavorable evolution after surgery for chronic sinusitis and nasal polyposis. YMHN. 2006; 134:991-6.

10. Ceri H, Olson ME, Stremick C, Read RR, Morck D, Buret A. The Calgary Biofilm Device: new technology for rapid determination of antibiotic susceptibilities of bacterial biofilms. J Clin Microbiol. 1999; 37:1771-6.

11. Desrosiers M, Bendouah Z, Barbeau J. Effectiveness of topical antibiotics on Staphylococcus aureus biofilm in vitro. Am J Rhinol. 2007; 21:149-53.

12. Jervis-Bardy J, Boase S, Psaltis A, Foreman A, Wormald P-J. A randomized trial of mupirocin sinonasal rinses versus saline in surgically recalcitrant staphylococcal chronic rhinosinusitis. Laryngoscope. 2012; 122:2148-53.

13. Smith SS, Evans CT, Tan BK, Chandra RK, Smith SB, Kern RC. National burden of antibiotic use for adult rhinosinusitis. J Allergy Clin Immunol. 2013; 132:1230-2.

14. Riley P, Moore D, Ahmed F, Sharif MO,
Worthington HV. Xylitol-containing products for preventing dental caries in children and adults. Riley P, editor. Cochrane Database Syst Rev. 2015; 3:CD010743.

15. Weissman JD, Fernandez F, Hwang PH. Xylitol nasal irrigation in the management of chronic rhinosinusitis: A pilot study. Laryngoscope. 2011; 121:2468-72.

16. Stepanović S, Vuković D, Hola V, Di Bonaventura G, Djukić S, Cirković l, et al. Quantification of biofilm in microtiter plates: overview of testing conditions and practical recommendations for assessment of biofilm production by staphylococci. APMIS. 2007; 115:891-9.

17. Alves FRF, Silva MG, Rôças IN, Siqueira JF. Biofilm biomass disruption by natural substances with potential for endodontic use. Braz Oral Res. 2013; 27:20-5.

18. Ha KR, Psaltis AJ, Butcher AR, Wormald P-J, Tan LW. In vitro activity of mupirocin on clinical isolates of Staphylococcus aureus and its potential implications in chronic rhinosinusitis. Laryngoscope. 2008; 118:53540.

19. Azarpazhooh A, Limeback H, Lawrence HP, Shah PS. Xylitol for preventing acute otitis media in children up to 12 years of age. Cochrane Database Syst Rev. 2011;11:CD007095.

20. Sakallioğlu Ö, Güvenç IA, CIngi C. Xylitol and its usage in ENT practice. J Laryngol Otol. 2014; 7:1-6.

21. Katsuyama M, Masako K, Ichikawa $H$, 
Hideyuki I, Ogawa S, Shigeyuki O, et al. A novel method to control the balance of skin microflora. Part 1. Attack on biofilm of Staphylococcus aureus without antibiotics. J Dermatol Sci. 2005; 3:197-205.

22. Zabner J, Seiler MP, Launspach JL, Karp PH, Kearney WR, Look DC, et al. The osmolyte xylitol reduces the salt concentration of airway surface liquid and may enhance bacterial killing. Proc Natl Acad Sci USA.; 2000; 97:11614-9.

23. Brown $\mathrm{CL}$, Graham SM, Cable BB, Ozer EA, Taft PJ, Zabner J. Xylitol enhances bacterial killing in the rabbit maxillary sinus. Laryngoscope. John Wiley \& Sons, Inc; 2004; 114:2021-4.

24. Miyasawa H, Iwami Y, Mayanagi H, Takahashi N. Xylitol inhibition of anaerobic acid production by Streptococcus mutans at various pH levels. Oral Microbiol Immunol. 2003; 18:215-9.
25. Badet C, Furiga A, Thébaud N. Effect of xylitol on an in vitro model of oral biofilm. Oral Health Prev Dent. 2008; 6:337-41.

26. Ammons MCB, Ward LS, Dowd S, James GA Combined treatment of Pseudomonas aeruginosa biofilm with lactoferrin and xylitol inhibits the ability of bacteria to respond to damage resulting from lactoferrin iron chelation. Int J Antimicrob Agents. 201; 37:316-23.

27. Ammons MCB, Ward LS, Fisher ST, Wolcott $R D$, James $G A$. In vitro susceptibility of established biofilms composed of a clinical wound isolate of Pseudomonas aeruginosa treated with lactoferrin and xylitol. Int J Antimicrob Agents. 2009; 33:230-6.

28. Smith JJ, Travis SM, Greenberg EP, Welsh MJ. Cystic fibrosis airway epithelia fail to kil bacteria because of abnormal airway surface fluid. Cell. 1996; 85:229-36.
Assoc. Prof Richard Douglas

Department of Surgery

The University of Auckland

Private Bag 92019

Auckland 1142

New Zealand

Tel: +64-9-923 9820

Fax: +64-9-377 9656

E-mail: richard.douglas@auckland. ac.nz

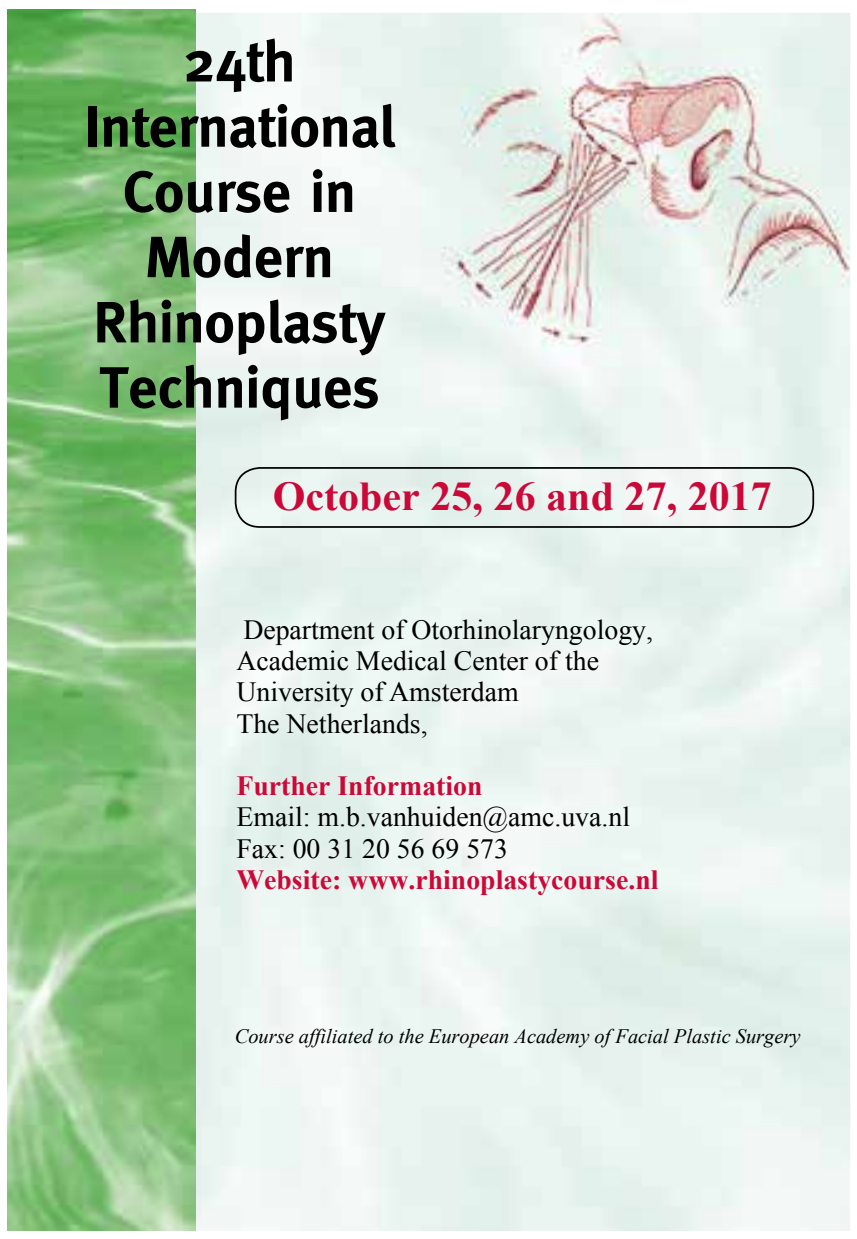

